\title{
Thermomagnetic corrections to $\pi-\pi$ scattering lengths in the linear sigma model
}

\author{
M. Loewe, ${ }^{1,2,3, *}$ E. Muñoz, ${ }^{1, \dagger}$ and R. Zamora ${ }^{4,5, \$}$ \\ ${ }^{1}$ Instituto de Física, Pontificia Universidad Católica de Chile, Casilla 306, Santiago 22, Chile \\ ${ }^{2}$ Centre for Theoretical and Mathematical Physics and Department of Physics, \\ University of Cape Town, Rondebosch 7700, South Africa \\ ${ }^{3}$ Centro Científico Tecnológico de Valparaíso-CCTVAL, Universidad Técnica Federico Santa María, \\ Casilla 110-V, Valparaíso, Chile \\ ${ }^{4}$ Instituto de Ciencias Básicas, Universidad Diego Portales, Casilla 298-V, Santiago, Chile \\ ${ }^{5}$ Centro de Investigación y Desarrollo de Ciencias Aeroespaciales (CIDCA), \\ Fuerza Aérea de Chile, Santiago 8020744, Chile
}

(Received 8 May 2019; published 9 December 2019)

\begin{abstract}
In this article, we extend our previous study of the $\pi-\pi$ scattering lengths under the presence of an external magnetic field, including finite temperature effects. The novelty of this work is precisely the introduction of temperature into the discussion and its interplay with the magnetic field. As in the previous article, we base our analysis in the linear sigma model, and our calculations are exact within this context. Although the effects are comparatively small, it is interesting to remark that the magnetic field and temperature display opposite effects over the scattering lengths.
\end{abstract}

DOI: 10.1103/PhysRevD.100.116006

\section{INTRODUCTION}

An interesting and relevant question in the field of heavy ion collision experiments is the search for the possibility of disentangling temperature and magnetic effects. A possible physical scenario to explore this problem is provided by $\pi-\pi$ scattering. The calculation of $\pi-\pi$ scattering lengths within Chiral perturbation theory (ChPT) was originally formulated in the classical paper by Gasser and Leutwyler [1], presenting explicit expressions at zero temperature and in the absence of magnetic field. Since then, higher-order calculations, including two-loop corrections in ChPT at zero temperature and in the absence of magnetic field have improved the estimation of those parameters as compared to experiment [2] (see also Table I for a comparison). In ChPT, also at $B=0$, finite temperature corrections to the phase shifts in $\pi$ - $\pi$ scattering are reported in [3], while explicit evaluation of the scattering lengths are presented in [4]. Finite temperature calculations of the $\pi-\pi$ scattering lengths in the framework of the Nambu-Jona-Lasinio (NJL) model are presented, in the absence of a magnetic

\footnotetext{
mloewe@fis.puc.cl

munozt@fis.puc.cl

†rzamorajofre@gmail.com
}

Published by the American Physical Society under the terms of the Creative Commons Attribution 4.0 International license. Further distribution of this work must maintain attribution to the author(s) and the published article's title, journal citation, and DOI. Funded by SCOAP. field, in [5], while the analysis of the $\rho \pi \pi$ vertex effect on residual pion scattering in the context of the extended-NJL model was discussed in [6], also in the absence of magnetic field. Despite its comparative simplicity, the linear sigma model (LSM) has been shown to provide reasonably accurate results for the scattering lengths as compared to experimental data [7] (see Table I for a comparison). Among the conceptual drawbacks of this approach is the ongoing controversy regarding the internal structure of the $f_{0}(550)$ resonance, which is treated as the massive $\sigma$ meson in LSM, neglecting the effects of its unknown internal degrees of freedom. A recent review and discussion of this subject are presented in [8]. On the other hand, from a phenomenological perspective, the LSM provides predictions of the $\pi-\pi$ scatttering lengths with similar or even better accuracy than standard one loop ChPT as compared to experiment [7] (see Table I). Moreover, recent further attempts to extend the LSM, by including vector mesons, showed that this leads to an effective low-energy action equivalent to ChPT [9].

Therefore, to explore the predictions of the LSM at finite temperature and a magnetic field in this context seems a necessary and, at least, academically interesting endeavor. The full interplay between finite temperature and a magnetic field is much less explored in the context of scattering lengths, but has nevertheless been recently considered, for instance, in the study of the evolution of the pion mass [12].

In this article, we will refer to $\pi-\pi$ scattering lengths, extending our previous results in LSM [13] at zero temperature and finite magnetic fields. In this work, we shall 
TABLE I. Comparison between the experimental values [10], first order prediction from chiral perturbation theory [11], and our results from linear sigma model at the three level [7].

\begin{tabular}{cccc}
\hline \hline & $\begin{array}{c}\text { Experimental } \\
\text { results }\end{array}$ & $\begin{array}{c}\text { Chiral perturbation } \\
\text { theory }\end{array}$ & $\begin{array}{c}\text { Linear sigma } \\
\text { model }\end{array}$ \\
\hline$a_{0}^{0}$ & $0.26 \pm 0.05$ & $\frac{7 m_{\pi}^{2}}{32 \pi f_{\pi}^{2}}=0.16$ & $\frac{10 m_{\pi}^{2}}{32 \pi f_{\pi}^{2}}=0.22$ \\
$b_{0}^{0}$ & $0.25 \pm 0.03$ & $\frac{m_{\pi}^{2}}{4 \pi f_{\pi}^{2}}=0.18$ & $\frac{49 m_{\pi}^{2}}{128 \pi f_{\pi}^{2}}=0.27$ \\
$a_{0}^{2}$ & $-0.028 \pm 0.0 .012$ & $-\frac{m_{\pi}^{2}}{16 \pi f_{\pi}^{2}}=-0.044$ & $-\frac{m_{\pi}^{2}}{16 \pi f_{\pi}^{2}}=-0.044$ \\
$b_{0}^{2}$ & $-0.082 \pm 0.008$ & $-\frac{m_{\pi}^{2}}{8 \pi f_{\pi}^{2}}=-0.089$ & $-\frac{m_{\pi}^{2}}{8 \pi f_{\pi}^{2}}=-0.089$ \\
$a_{1}^{1}$ & $0.038 \pm 0.002$ & $\frac{m_{\pi}^{2}}{24 \pi f_{\pi}^{2}}=0.030$ & $\frac{m_{\pi}^{2}}{24 \pi f_{\pi}^{2}}=0.030$ \\
$b_{1}^{1}$ & $\ldots$ & 0 & $\frac{m_{\pi}^{2}}{48 \pi f_{\pi}^{2}}=0.015$ \\
\hline \hline
\end{tabular}

present analytical results that fully capture both thermal and magnetic effects without any restriction or special ordering in these parameters. For this purpose, our calculations will be based on the linear sigma model using techniques associated to the spectral density functions that allows us to handle the infinite series over Landau levels in a closed analytical form. Infinite Matsubara sums were also performed exactly with complex contour integration techniques.

The paper is organized as follows: In Sec. II, we introduce the linear sigma model and the general formalism for scattering lengths. In Sec. III, the detailed Feynman diagrams are displayed, using the Schwinger propagators for finite magnetic fields. The two master integrals required for the calculation of all the diagrams are formulated. Therein, we also display the analytical results for the scattering lengths, with mathematical details deferred to the Appendixes. Finally, in Sec. IV, we show our numerical results, discussing the combined effects of temperature and a magnetic field.

\section{LINEAR SIGMA MODEL AND $\pi$ - $\pi$ SCATTERING}

In our previous article [13], we studied the $\pi-\pi$ scattering lengths within the context of the linear sigma model (LSM) in the phase where the chiral symmetry is broken,

$$
\begin{aligned}
\mathcal{L}= & \bar{\psi}\left[i \gamma^{\mu} \partial_{\mu}-m_{\psi}-g\left(\sigma+i \vec{\pi} \cdot \vec{\tau} \gamma_{5}\right)\right] \psi \\
& +\frac{1}{2}\left[(\partial \vec{\pi})^{2}+m_{\pi}^{2} \vec{\pi}^{2}\right]+\frac{1}{2}\left[(\partial \sigma)^{2}+m_{\sigma}^{2} \sigma^{2}\right] \\
& -\lambda^{2} v \sigma\left(\sigma^{2}+\vec{\pi}^{2}\right)-\frac{\lambda^{2}}{4}\left(\sigma^{2}+\vec{\pi}^{2}\right)^{2}+\left(\varepsilon c-v m_{\pi}^{2}\right) \sigma .
\end{aligned}
$$

In the expression above, $v=\langle\sigma\rangle$ is the vacuum expectation value of the original scalar field $\sigma$. This expression emerges after expanding the sigma field around $v . \varepsilon c \sigma$ is the term that breaks explicitly the $S U(2) \times S U(2)$ chiral symmetry, and $\varepsilon$ is a small dimensionless parameter. The model includes a doublet of Fermi fields, which in our case will be ignored since they represent nucleons which are too heavy as compared with the scalar sigma meson and the relevant energy scale. It is straightforward to see that $m_{\psi}=g v, m_{\pi}^{2}=\mu^{2}+\lambda^{2} v^{2}$, and $m_{\sigma}^{2}=\mu^{2}+3 \lambda^{2} v^{2}$. For details, see [14]. As displayed in Table I, the predictions for the scattering lengths calculated in the LSM compare very well with experimental data and, moreover, display a better agreement than those arising from chiral perturbation theory at the tree level.

Perturbation theory at the tree level allows us to identify the pion decay constants as $f_{\pi}=v$. Finite temperature effects on this model have been studied by several authors, discussing the thermal evolution of masses, $f_{\pi}(T)$, the effective potential, etc., [12,15-24].

The most general decomposition for the scattering amplitude for particles with definite isospin quantum numbers is $[25,26]$

$$
\begin{aligned}
T_{\alpha \beta ; \delta \gamma}= & A(s, t, u) \delta_{\alpha \beta} \delta_{\delta \gamma}+A(t, s, u) \delta_{\alpha \gamma} \delta_{\beta \delta} \\
& +A(u, t, s) \delta_{\alpha \delta} \delta_{\beta \gamma},
\end{aligned}
$$

where $\alpha, \beta, \gamma, \delta$ denote isospin components.

By using appropriate projection operators, it is possible to find the following isospin dependent scattering amplitudes:

$$
\begin{gathered}
T^{0}=3 A(s, t, u)+A(t, s, u)+A(u, t, s), \\
T^{1}=A(t, s, u)-A(u, t, s), \\
T^{2}=A(t, s, u)+A(u, t, s),
\end{gathered}
$$

where $T^{I}$ denotes a scattering amplitude in a given isospin channel $I=\{0,1,2\}$.

As it is well known [26], below the inelastic threshold any scattering amplitude can be expanded in terms of partial amplitudes which can be parametrized by the phase shifts for each angular momentum channel $\ell$. Therefore, in the low-energy region, the isospin dependent scattering amplitude can be expanded in partial wave components $T_{\ell}^{I}$. The real part of this amplitude,

$$
\Re\left(T_{\ell}^{I}\right)=\left(\frac{p^{2}}{m_{\pi}^{2}}\right)^{\ell}\left(a_{\ell}^{I}+\frac{p^{2}}{m_{\pi}^{2}} b_{\ell}^{I}+\cdots\right)
$$

is expressed in terms of the scattering lengths $a_{\ell}^{I}$, and the scattering slopes $b_{\ell}^{I}$, respectively. The scattering lengths satisfy the hierarchy $\left|a_{0}^{I}\right|>\left|a_{1}^{I}\right|>\left|a_{2}^{I}\right| \cdots$. In particular, in order to obtain the scattering lengths $a_{0}^{I}$, it is sufficient to calculate the scattering amplitude $T^{I}$ in the static limit, i.e., when $s \rightarrow 4 m_{\pi}^{2}, t \rightarrow 0$, and $u \rightarrow 0$,

$$
a_{0}^{I}=\frac{1}{32 \pi} T^{I}\left(s \rightarrow 4 m_{\pi}^{2}, t \rightarrow 0, u \rightarrow 0\right) .
$$

The first measurement of $\pi-\pi$ scattering lengths was carried on by Rosellet et al. [27]. More recently, these parameters have been measured using pionium atoms in the DIRAC 
experiment [28] and also through the decay of heavy quarkonium states into $\pi$ - $\pi$ final states where the so-called cusp effect was found [29]. Independent measurements of these parameters were carried on by the NA48/2 Collaboration [30] as well as by the BNL-E865 Collaboration [31] using Ke4 decays.

In our recent work [13], we obtained an exact analytical expression for Eq. (7) for $I=0,2$ in a background magnetic field of arbitrary strength, at zero temperature. In this article, we extend our previous result to include finite temperatures. Therefore, in what follows, we shall present the exact expression for the scattering lengths Eq. (7) at arbitrary magnitudes of the external magnetic field and temperature.

\section{SCATTERING LENGTHS AT FINITE MAGNETIC FIELD AND FINITE TEMPERATURE}

In a previous work, based on a perturbative treatment of the bosonic Schwinger propagator valid for small magnetic fields, some of us discussed the magnetic dependence of the $\pi-\pi$ scattering lengths within the context of the linear sigma model [32]. We found that this magnetic evolution displays an opposite trend with respect to thermal corrections on the scattering lengths, in agreement with the literature [33]. At low magnetic field intensities, the scattering lengths in the isospin channel $I=2$ increase, whereas their projection into the channel $I=0$ diminishes, both as functions of the magnetic field. More recently, we extended the analysis in the zero temperature scenario, thus obtaining exact analytical expressions valid in the whole range of magnetic field intensities [13].

In the linear sigma model, the relevant diagrams that contribute to $\pi$ - $\pi$ scattering are shown in Fig. 1. Notice that tadpolelike diagrams associated to mass corrections of the sigma field do not contribute to the $\pi-\pi$ scattering amplitudes, since their imaginary part vanishes. These tadpoles are extremely small in the limit of a very large mass of the sigma field. This approximation is valid since, as we know, $m_{\sigma} \approx 550 \mathrm{MeV}$ is much larger than the pion mass. Fermions, i.e., nucleons that may interact with pions, are not considered in our discussion. As a consequence, the sigma field propagator is contracted to a point.

From these considerations, we see that all relevant diagrams reduce to a horizontal ( $s$ channel) or vertical ( $t$ and $u$ channels) "fish-type" pion loops contributions, as shown in Fig. 2. Then, we need to compute such diagrams as a function of the magnetic field intensity and finite temperature. This is an interesting problem, not only because of physical implications, but also due to new analytical results that we shall present below.

Let us derive our starting expression for the bosonic propagator as a sum of Landau levels [13,34]. The bosonic Schwinger propagator for a charged pion of charge $q$ subject to a uniform magnetic field along the third spatial coordinate in the proper time representation is given by

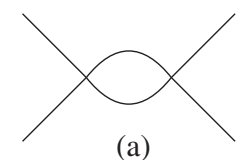

(a)

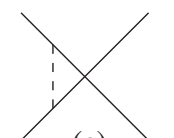

(c)

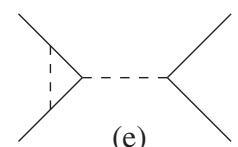

(e)

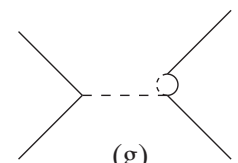

(g)

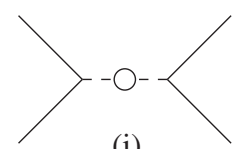

(i)

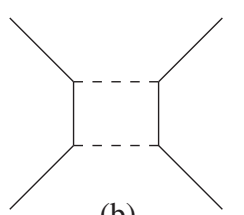

(b)

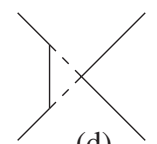

(d)

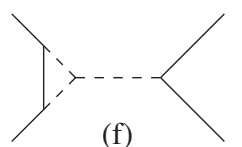

(f)

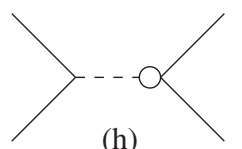

(h)

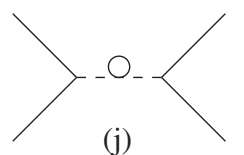

(j)
FIG. 1. One-loop diagrams relevant to the $\pi-\pi$ scattering lengths. Continuous and dashed lines represent pions and $\sigma$ mesons, respectively.

$$
i D^{B}(k)=\int_{0}^{\infty} \frac{d s}{\cos (q B s)} e^{i s\left(k_{\|}^{2}-k_{\perp}^{2} \frac{\tan (q B s)}{q B s}-m_{\pi}^{2}+i \epsilon\right)} .
$$

After inserting this propagator in the fish-type diagrams, we get

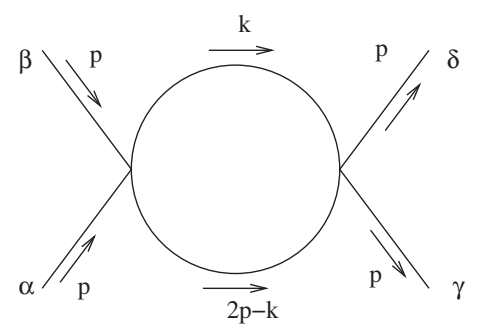

(a) s-channel diagram.

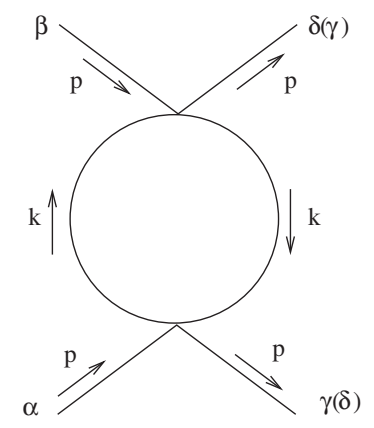

(b) $t$ and $u$ channel diagram.

FIG. 2. "Fish-type" diagrams. (a) $s$-channel diagram. (b) $t$ and $u$ channel diagram. 


$$
\begin{gathered}
I_{a}[B, p]=\int \frac{d^{4} k}{(2 \pi)^{4}} i D^{B}(k) i D^{B}(k-2 p), \\
I_{b}[B]=\int \frac{d^{4} k}{(2 \pi)^{4}}\left[i D^{B}(k)\right]^{2} .
\end{gathered}
$$

In order to calculate the scattering amplitude, we work in the center-of-mass momentum $p=\left(2 p_{0}, \overrightarrow{0}\right)$,

$$
\begin{gathered}
I_{1}\left[B, p_{0}\right]=\int \frac{d^{4} k}{(2 \pi)^{4}} i D^{B}\left(k_{0}, \mathbf{k}\right) i D^{B}\left(k_{0}-2 p_{0}, \mathbf{k}\right), \\
I_{2}[B]=\int \frac{d^{4} k}{(2 \pi)^{4}}\left[i D^{B}\left(k_{0}, \mathbf{k}\right)\right]^{2} .
\end{gathered}
$$

Note that all the diagrams displayed in Fig. 1 reduce, in the approximation of a very massive sigma field, to either one of the integrals defined by Eqs. (11) and (12). Symmetry factors, isospin index contractions, and multiplicity factors should be included, however, for each diagram.

For technical purposes, we shall calculate the integrals with the expression for the propagator at finite magnetic field in terms of Landau levels, as presented in [34],

$$
i D^{B}(k)=2 \sum_{l=0}^{\infty}(-1)^{l} L_{l}\left(\frac{2 k_{\perp}^{2}}{q B}\right) e^{-k_{\perp}^{2} / q B} i \Delta_{l}^{B}\left(k_{\|}\right),
$$

where $L_{l}(z)$ are the Laguerre polynomials, and where we have defined the effective "parallel" propagators,

$$
i \Delta_{l}^{B}\left(k_{\|}\right)=\frac{i}{k_{\|}^{2}-(2 l+1) q B-m_{\pi}^{2}+i \epsilon} .
$$

The above expressions correspond to the zero-temperature scenario. However, it is straightforward to generalize them to the finite temperature case by analytic continuation into Matsubara frequency space, i.e.,

$$
\begin{array}{ll}
k_{0} \rightarrow i \omega_{n}=2 \pi n / \beta, & n \in Z, \\
p_{0} \rightarrow i \nu_{m}=2 \pi m / \beta, & m \in Z,
\end{array}
$$

where $\beta=1 / T$, and the corresponding substitution of the integral in $k_{0}$ by a sum,

$$
\int \frac{d^{2} k_{\|}}{(2 \pi)^{2}} \rightarrow \frac{i}{\beta} \sum_{n \in Z} \int_{-\infty}^{+\infty} \frac{d k_{3}}{2 \pi}
$$

Let us first consider the calculation of $I_{1}\left[T, B, p_{0}\right]$. After its definition in Eq. (12), substituting the infinite series for the propagators, Eq. (13), we are lead to

$$
\begin{aligned}
I_{1}\left[T, B, i \nu_{m}\right]= & \frac{i}{\beta} \sum_{n \in Z} \int \frac{d^{3} k}{(2 \pi)^{3}} i D^{B}\left(i \omega_{n}, \mathbf{k}\right) \\
& \times i D^{B}\left(i \omega_{n}-2 i \nu_{m}, \mathbf{k}\right) \\
= & 4 \sum_{l=0}^{\infty} \sum_{l^{\prime}=0}^{\infty}(-1)^{l+l^{\prime}} G_{l, l^{\prime}}\left(i \nu_{m}\right) \\
& \times\left[\int \frac{d^{2} k_{\perp}}{(2 \pi)^{2}} e^{-2 k_{\perp}^{2} / q B} L_{l}\left(\frac{2 k_{\perp}^{2}}{q B}\right) L_{l^{\prime}}\left(\frac{2 k_{\perp}^{2}}{q B}\right)\right] .
\end{aligned}
$$

Here, we have defined the functions,

$$
\begin{aligned}
G_{l, l^{\prime}}\left(T, i \nu_{m}\right)= & \int_{-\infty}^{\infty} \frac{d k_{3}}{2 \pi} \frac{i}{\beta} \sum_{n \in Z} i \Delta_{l}^{B}\left(i \omega_{n}, k_{3}\right) \\
& \times i \Delta_{l^{\prime}}^{B}\left(i \omega_{n}-2 i \nu_{m}, k_{3}\right) .
\end{aligned}
$$

Let us now calculate the integral over the Laguerre polynomials in the second term, by using two-dimensional "spherical coordinates", with $0 \leq\left|k_{\perp}\right|<\infty$,

$$
d^{2} k_{\perp}=2 \pi\left|k_{\perp}\right| d\left|k_{\perp}\right|=\frac{\pi q B}{2} d x
$$

where we have defined the auxiliary variable $x=2 k_{\perp}^{2} / q B$, with $0 \leq x<\infty$. Therefore, we have

$$
\begin{aligned}
& \int \frac{d^{2} k_{\perp}}{(2 \pi)^{2}} e^{-2 k_{\perp}^{2} / q B} L_{l}\left(\frac{2 k_{\perp}^{2}}{q B}\right) L_{l^{\prime}}\left(\frac{2 k_{\perp}^{2}}{q B}\right) \\
& =\frac{1}{4 \pi^{2}} \frac{\pi q B}{2} \int_{0}^{\infty} d x e^{-x} L_{l}(x) L_{l^{\prime}}(x) \\
& =\frac{q B}{8 \pi} \delta_{l, l^{\prime}},
\end{aligned}
$$

where the orthogonality relation between Laguerre polynomials was used. Substituting this result into Eq. (17), we end up with the expression,

$$
I_{1}\left[T, B, i \nu_{m}\right]=\frac{q B}{2 \pi} \sum_{l=0}^{\infty} G_{l, l}\left(T, i \nu_{m}\right) .
$$

As shown in detail in the Appendix A, we calculate $G_{l, l}\left(i \nu_{m}\right)$ by first integrating over $k_{0}$ in the complex plane and later over $k_{3}$. This procedure allows us to obtain the infinite series,

$$
\begin{aligned}
I_{1}\left[T, B, i \nu_{m}\right]= & \frac{q B}{4 \pi\left(i \nu_{m}\right)} \\
& \times \Im m \sum_{l=0}^{\infty} \int_{-\infty}^{+\infty} \frac{d k_{3}}{2 \pi} g\left(T, E_{l}\left(k_{3}\right), i \nu_{m}\right),
\end{aligned}
$$

with $E_{l}\left(k_{3}\right)=\sqrt{k_{3}^{2}+m_{\pi}^{2}+q B(2 l+1)}$, and the functions $g\left(T, E, i \nu_{m}\right)$ defined by 


$$
g\left(T, E, i \nu_{m}\right)=\frac{\operatorname{coth}(\beta E / 2)}{E\left[E-i \nu_{m}\right]} .
$$

As discussed in the Appendix B, we introduce the spectral density,

$$
\rho(E)=\sum_{l=0}^{\infty} \int_{-\infty}^{\infty} \frac{d k_{3}}{2 \pi} \delta\left(E-E_{l}\left(k_{3}\right)\right),
$$

such that Eq. (22) can be expressed as single integral over the energy domain,

$I_{1}\left[T, B, i \nu_{m}\right]=\frac{q B}{4 \pi\left(i \nu_{m}\right)} \Im m \int_{0}^{\infty} d E \rho(E) g\left(T, E, i \nu_{m}\right)$,

where a closed analytical expression for the spectral density was derived in the Appendix B,

$$
\begin{aligned}
\rho(E)= & \frac{E}{\pi \sqrt{2 q B}} \Theta\left(E-\sqrt{m_{\pi}^{2}+q B}\right) \\
& \times\left\{\zeta\left(\frac{1}{2}, \frac{E^{2}-m_{\pi}^{2}-q B}{2 q B}-\left\lfloor\frac{E^{2}-m_{\pi}^{2}-q B}{2 q B}\right\rfloor\right)\right. \\
& \left.-\zeta\left(\frac{1}{2}, \frac{E^{2}-m_{\pi}^{2}-q B}{2 q B}+1\right)\right\},
\end{aligned}
$$

with $\lfloor z\rfloor$ the integer part of $z$ and $\zeta(s, z)$ the Hurwitz Zeta function. In order to obtain the scattering lengths $a_{0}^{I}$, we use the decomposition of the scattering amplitude in the different isospin channels presented in Sec. II. Since we are only interested in the scattering lengths $a_{0}^{I}$, it is enough to calculate the scattering amplitude in the static limit. In this way, we obtain

$a_{0}^{0}(T, B)=a_{0}^{0}+\frac{1}{32 \pi}(3 A(s, t, u)+A(t, s, u)+A(u, t, s))$,

$a_{0}^{2}(T, B)=a_{0}^{2}+\frac{1}{32 \pi}(A(t, s, u)+A(u, t, s))$.

For our analysis, we normalize by the values at tree level from the linear sigma model $\left(a_{0}^{0}=0.22\right.$ and $a_{0}^{2}=$ -0.044 [7]). Here, $A(s, t, u), A(t, s, u)$, and $A(u, t, s)$ correspond to all $s$-channel, $t$-channel, and $u$-channel contributions, respectively. On the other hand, the $s$-channel contribution is obtained from $I_{1}\left[T, B, i \nu_{m} \rightarrow p_{0}=m_{\pi}\right]$, while those for the $t$ and $u$ channels are obtained from $I_{2}[T, B]=I_{1}\left[T, B, i \nu_{m} \rightarrow p_{0}=0\right]$, according to the following expressions:

$$
\begin{aligned}
A(s, t, u)= & -4 \lambda^{4}\left(1-\frac{12 \lambda^{2} v^{2}}{m_{\sigma}^{2}}+\frac{24 \lambda^{4} v^{4}}{m_{\sigma}^{4}}\right) \\
& \times I_{1}\left[T, B, m_{\pi}\right], \\
A(t, s, u)+A(u, t, s)= & -8 \lambda^{4}\left(1-\frac{12 \lambda^{2} v^{2}}{m_{\sigma}^{2}}+\frac{24 \lambda^{4} v^{4}}{m_{\sigma}^{4}}\right) \\
& \times I_{1}[T, B, 0] .
\end{aligned}
$$

The mass for the sigma meson is set to $m_{\sigma}=550 \mathrm{MeV}$, and the mass for the pion is set to $m_{\pi}=140 \mathrm{MeV}$, with the parameter $v=89 \mathrm{MeV}$ and $\lambda^{2}=4.26$.

\section{RESULTS AND CONCLUSIONS}

We have presented a novel method to calculate the scattering lengths for $\pi-\pi$ scattering within the linear sigma model at the one-loop level, in the isospin channels $I=\{0,2\}$, as functions of the temperature and external magnetic field intensity. We display the calculated scattering lengths $a_{0}^{0}$ and $a_{0}^{2}$ as a function of temperature for three different magnetic field values in Figs. 3-5. From the three figures, it is clear that at a finite magnetic field, while $a_{0}^{0}$ decreases as a function of temperature, $a_{0}^{2}$ displays the opposite trend. It is interesting to remark that already the presence of a small magnetic field is sufficient to invert

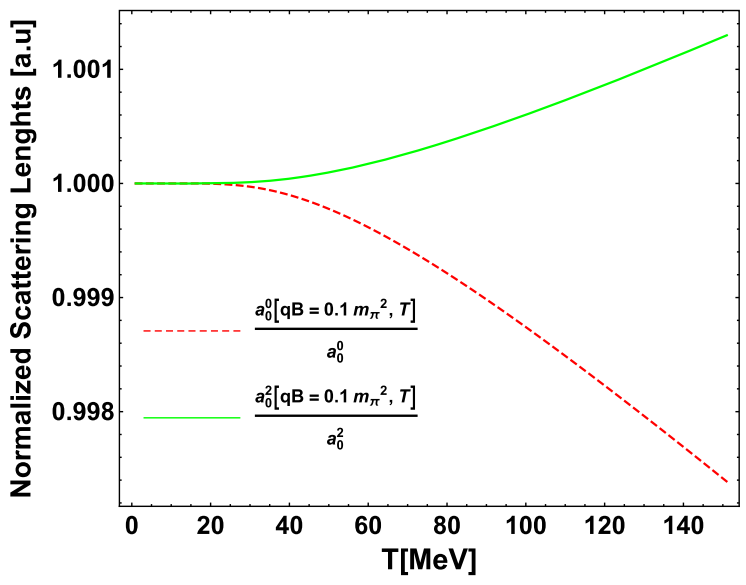

FIG. 3. The scattering parameters $a_{0}^{0}(B, T) / a_{0}^{0}$ (dashed) and $a_{0}^{2}(B, T) / a_{0}^{2}$ (solid) are displayed as a function of temperature for a magnetic field of $q B=0.1 m_{\pi}^{2}$.

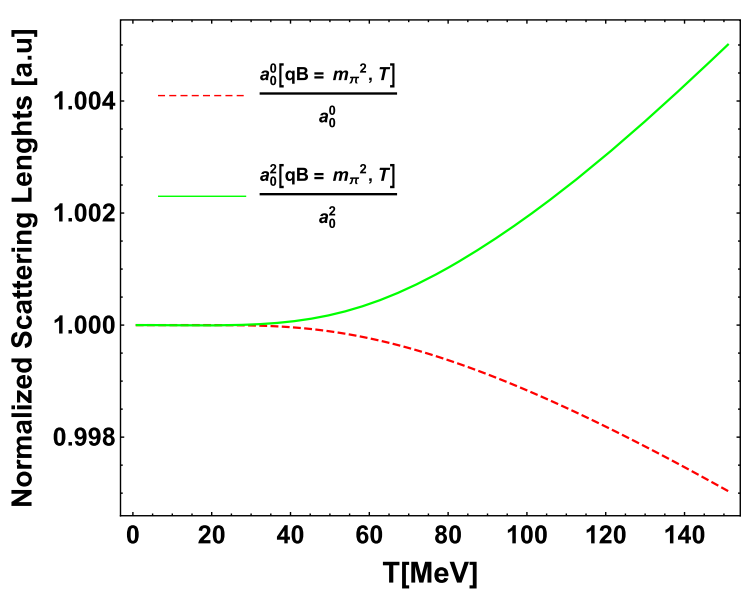

FIG. 4. The scattering parameters $a_{0}^{0}(B, T) / a_{0}^{0}$ (dashed) and $a_{0}^{2}(B, T) / a_{0}^{2}$ (solid) are displayed as a function of temperature for a magnetic field of $q B=m_{\pi}^{2}$. 


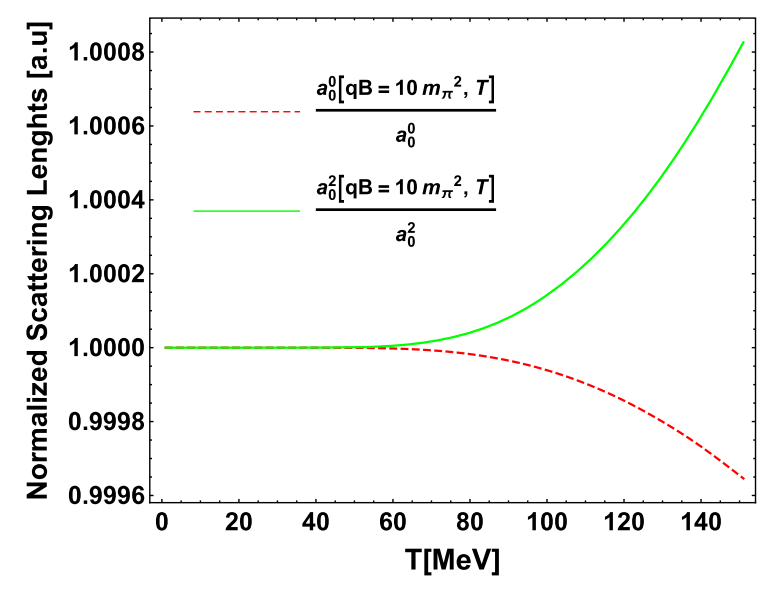

FIG. 5. The scattering parameters $a_{0}^{0}(B, T) / a_{0}^{0}$ (dashed) and $a_{0}^{2}(B, T) / a_{0}^{2}$ (solid) are displayed as a function of temperature for a magnetic field of $q B=10 m_{\pi}^{2}$.

the temperature dependence at a zero magnetic field [33,35]. This effect can be explained by the symmetry of the scattering length parameters, where $a_{0}^{0}$ arises from the trace of the tensor, which is a scalar, whereas the isospin 2 channel corresponds to the most symmetric realization. Qualitatively, the magnetic moment of the semiclassical orbits associated to the Landau levels tend to align along the magnetic field direction, while temperature tends to randomize those directions. Therefore, under finite magnetic fields, the most symmetric state enhances its scattering rate.

\section{ACKNOWLEDGMENTS}

M. L. acknowledges support from FONDECYT (Chile) under Grants No. 1170107 and No. 1190192 and ConicytPIA/BASAL (Chile) Grant No. FB0821. M. L. would also like to thank Marc Knecht for useful comments about the measurements by the NA48/2 and the BNL-E865 Collaborations. E. Muñoz acknowledges support from FONDECYT under Grant No. 1190361 and R. Z. would like to thank support from CONICYT FONDECYT Iniciación under Grant No. 11160234 and FONDECYT No. 1170107 and No. 1190192.

\section{APPENDIX A: MATSUBARA SUMS AND INTEGRALS OVER $\boldsymbol{k}_{3}$}

Here, we present in detail the calculation of the Matsubara sums involved in Eq. (22) of the main text. Using the definition of the "parallel" propagators Eq. (14), we have

$$
\begin{aligned}
G_{l, l}\left(T, i \nu_{m}\right)= & \int \frac{d k_{3}}{2 \pi} \frac{i}{\beta} \sum_{n \in Z} i \Delta_{l}^{B}\left(i \omega_{n}, k_{3}\right) \\
& \times i \Delta_{l}^{B}\left(i \omega_{n}-2 i \nu_{m}, k_{3}\right) \\
= & i \frac{i^{2}}{2 \pi} \int_{-\infty}^{+\infty} d k_{3} f\left(T, E_{l}\left(k_{3}\right), i \nu_{m}\right),
\end{aligned}
$$

where we have defined the expression,

$$
f\left(T, E_{l}\left(k_{3}\right), i \nu_{m}\right)=\frac{1}{\beta} \sum_{n \in Z} \frac{1}{A\left(i \omega_{n}, k_{3}\right) C\left(i \omega_{n}, k_{3}\right)},
$$

with

$$
\begin{aligned}
& A\left(i \omega_{n}, k_{3}\right)=\left(i \omega_{n}\right)^{2}-E_{l}\left(k_{3}\right)^{2}+i \epsilon, \\
& C\left(i \omega_{n}, k_{3}\right)=\left(i \omega_{n}-2 i \nu_{m}\right)^{2}-E_{l}\left(k_{3}\right)^{2}+i \epsilon,
\end{aligned}
$$

and $E_{l}\left(k_{3}\right)=\sqrt{k_{3}^{2}+m_{\pi}^{2}+q B(2 l+1)}$. The Matsubara sum can be evaluated, as usual, by constructing a contour integral on the complex $k_{0}$ plane,

$$
\oint_{C \oplus C_{1} \oplus C_{2} \oplus C_{3} \oplus C_{4}} d k_{0} \frac{n_{B}\left(k_{0}\right)}{A\left(k_{0}, k_{3}\right) C\left(k_{0}, k_{3}\right)},
$$

where the contour is depicted in Fig. (6). Here, the integrand contains as a factor the Bose-Einstein distribution,

$$
n_{B}\left(k_{0}\right)=\left(\exp \left(\beta k_{0}\right)-1\right)^{-1}
$$

that possesses infinitely many simple poles at the Matsubara frequencies $k_{0}=i \omega_{n}=i 2 \pi n / \beta$, with residue,

$$
\lim _{k_{0} \rightarrow i \omega_{n}} \frac{k_{0}-i \omega_{n}}{e^{i \omega_{n}} \exp \left(\beta\left(k_{0}-i \omega_{n}\right)\right)-1}=\frac{1}{\beta} .
$$

In addition, the integrand in Eq. (A4) possesses four simple poles at $k_{0}^{(1,2)}= \pm E_{l}\left(k_{3}\right) \mp i \epsilon^{\prime}$ and $k_{0}^{(3,4)}=2 i \nu_{m} \pm E_{l}\left(k_{3}\right) \mp$ $i \epsilon^{\prime}$, i.e., two of them located on the positive imaginary plane, while the other two are located on the negative imaginary plane. By direct application of the residue theorem, we have

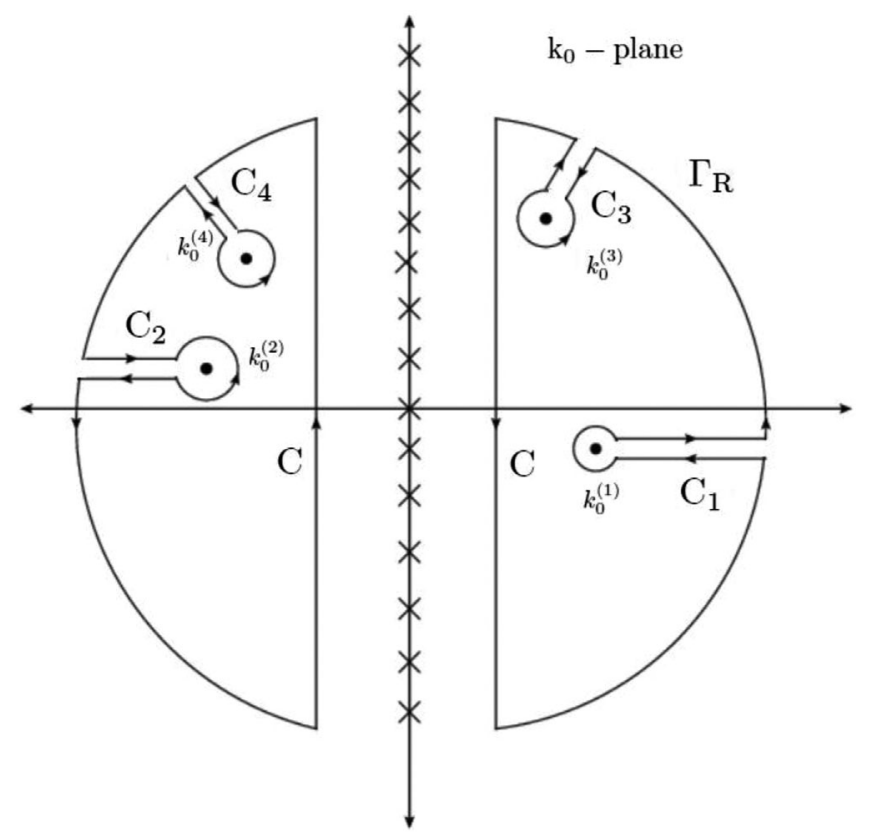

FIG. 6. Integration contour in the complex $k_{0}$ plane. 


$$
\begin{aligned}
& \oint d k_{0} \frac{n_{B}\left(k_{0}\right)}{A\left(k_{0}, k_{3}\right) C\left(k_{0}, k_{3}\right)} \\
& =0=2 \pi i\left(\sum_{j=1}^{4} n_{B}\left(k_{0}^{(j)}\right) \lim _{k_{0} \rightarrow k_{0}^{(j)}} \frac{\left(k_{0}-k_{0}^{(j)}\right)}{A\left(k_{0}, k_{3}\right) C\left(k_{0}, k_{3}\right)}\right. \\
& \left.\quad+\frac{1}{\beta} \sum_{n \in Z} \frac{1}{A\left(i \omega_{n}, k_{3}\right) C\left(i \omega_{n}, k_{3}\right)}\right) .
\end{aligned}
$$

Clearly, the second term within the parenthesis is precisely the function $f_{l}\left(T, k_{3}, i \nu_{m}\right)$ defined in Eq. (A2), and hence, we have

$$
\begin{aligned}
f\left(T, E_{l}\left(k_{3}\right), i \nu_{m}\right)= & -\sum_{j=1}^{4} n_{B}\left(k_{0}^{(j)}\right) \lim _{k_{0} \rightarrow k_{0}^{(j)}} \frac{\left(k_{0}-k_{0}^{(j)}\right)}{A\left(k_{0}, k_{3}\right) C\left(k_{0}, k_{3}\right)} \\
= & \frac{\left[n_{B}\left(E_{l}\left(k_{3}\right)\right)-n_{B}\left(-E_{l}\left(k_{3}\right)\right)\right]}{4 i \nu_{m} E_{l}\left(k_{3}\right)} \\
& \times\left\{\frac{1}{2 E_{l}\left(k_{3}\right)-2 i \nu_{m}}-\frac{1}{2 E_{l}\left(k_{3}\right)+2 i \nu_{m}}\right\},
\end{aligned}
$$

where we used the elementary property of the Bose distribution $n_{B}\left(z+i \nu_{m}\right)=n_{B}(z)$. Moreover, we also have $n_{B}(-z)=-1-n_{B}(z)$. Therefore, $n_{B}\left(E_{l}\left(k_{3}\right)\right)-$ $n_{B}\left(-E_{l}\left(k_{3}\right)=2 n_{B}\left(E_{l}\left(k_{3}\right)\right)+1=\operatorname{coth}\left(\beta E_{l}\left(k_{3}\right) / 2\right)\right.$, and hence, we finally obtain

$$
\begin{aligned}
f\left(T, E_{l}\left(k_{3}\right), i \nu_{m}\right)= & \frac{\operatorname{coth}\left(\beta E_{l}\left(k_{3}\right) / 2\right)}{8 i \nu_{m} E_{l}\left(k_{3}\right)} \\
& \times\left\{\frac{1}{E_{l}\left(k_{3}\right)-i \nu_{m}}-\frac{1}{E_{l}\left(k_{3}\right)+i \nu_{m}}\right\} .
\end{aligned}
$$

Let us define the functions,

$$
g\left(T, E, i \nu_{m}\right)=\frac{\operatorname{coth}(\beta E / 2)}{E\left[E-i \nu_{m}\right]},
$$

such that

$$
\begin{aligned}
f\left(T, E_{l}\left(k_{3}\right), i \nu_{m}\right)= & \frac{1}{8 i \nu_{m}}\left[g\left(T, E_{l}\left(k_{3}\right), i \nu_{m}\right)\right. \\
& \left.-g\left(T, E_{l}\left(k_{3}\right),-i \nu_{m}\right)\right] .
\end{aligned}
$$

Now we calculate the integral over $k_{3}$. Inserting Eq. (A8) into Eq. (A1), we have

$$
\begin{aligned}
G_{l, l}\left(T, i \nu_{m}\right)= & -\frac{i}{8 i \nu_{m}} \int_{-\infty}^{\infty} \frac{d k_{3}}{2 \pi}\left\{g\left(T, E_{l}\left(k_{3}\right), i \nu_{m}\right)\right. \\
& \left.-g\left(T, E_{l}\left(k_{3}\right),-i \nu_{m}\right)\right\} \\
= & \frac{1}{4 i \nu_{m}} \Im m \int_{-\infty}^{\infty} \frac{d k_{3}}{2 \pi} g\left(T, E_{l}\left(k_{3}\right), i \nu_{m}\right) .
\end{aligned}
$$

\section{APPENDIX B: SPECTRAL DENSITY}

As defined in the main text, here we present the analytical calculation for the spectral density $\rho(E)$. By definition, we have

$$
\rho(E)=\sum_{l=0}^{\infty} \int_{-\infty}^{\infty} \frac{d k_{3}}{2 \pi} \delta\left(E-E_{l}\left(k_{3}\right)\right)
$$

The condition enforced by the argument of the delta function possesses two algebraic solutions, i.e., $k_{3}=$ $\pm \sqrt{E^{2}-m_{\pi}^{2}-q B(2 l+1)}$, but only the positive one possesses support under the integration domain,

$$
\begin{aligned}
\delta\left(E-E_{l}\left(k_{3}\right)\right)= & \frac{E}{\left|k_{3}\right|}\left[\delta\left(k_{3}+\sqrt{E^{2}-m_{\pi}^{2}-q B(2 l+1)}\right)\right. \\
& \left.+\delta\left(k_{3}-\sqrt{E^{2}-m_{\pi}^{2}-q B(2 l+1)}\right)\right] .
\end{aligned}
$$

By carrying out the integration explicitly, we obtain the expression,

$$
\rho(E)=\frac{E}{\pi} \sum_{l=0}^{\infty} \frac{\Theta\left(E-\sqrt{m_{\pi}^{2}+q B(2 l+1)}\right)}{\sqrt{E^{2}-m_{\pi}^{2}-q B(2 l+1)}} .
$$

The infinite sum can be calculated, after noticing that the Heaviside step function restricts the upper limit of the Landau level $0 \leq l \leq l_{\max }(E)$ for each given value of the energy, through the condition,

$$
l_{\max }(E)=\left\lfloor\frac{E^{2}-m_{\pi}^{2}-q B}{2 q B}\right\rfloor,
$$

with $\lfloor z\rfloor$ the integer part of $z$. Therefore, from this definition, the analytical expression for the spectral density becomes

$$
\begin{aligned}
\rho(E)= & \frac{E}{\pi \sqrt{2 q B}} \Theta\left(E-\sqrt{m_{\pi}^{2}+q B}\right) \\
& \times \sum_{l=0}^{l_{\max }(E)} \frac{1}{\sqrt{\frac{E^{2}-m_{\pi}^{2}-q B}{2 q B}-l}} .
\end{aligned}
$$

It is now convenient to organize the finite sum differently, by defining $\bar{l}=l_{\max }(E)-l$. Clearly, we have $0 \leq \bar{l} \leq$ $l_{\max }(E)$. Therefore, the expression above can be written in the equivalent form,

$$
\begin{aligned}
\rho(E)= & \frac{E}{\pi \sqrt{2 q B}} \Theta\left(E-\sqrt{m_{\pi}^{2}+q B}\right) \\
& \times \sum_{\bar{l}=0}^{l_{\max }(E)} \frac{1}{\sqrt{\frac{E^{2}-m_{\pi}^{2}-q B}{2 q B}-l_{\max }(E)+\bar{l}}} .
\end{aligned}
$$


By applying the identity that follows from the definition of the Hurwitz Zeta function, for $n \in \mathbb{N}_{0}$,

$$
\sum_{l=0}^{n} \frac{1}{(z+l)^{s}}=\zeta(s, z)-\zeta(s, z+n+1)
$$

we can reduce the density of states to the analytical expression,

$$
\begin{aligned}
\rho(E)= & \frac{E}{\pi \sqrt{2 q B}} \Theta\left(E-\sqrt{m_{\pi}^{2}+q B}\right) \\
& \times\left\{\zeta\left(\frac{1}{2}, \frac{E^{2}-m_{\pi}^{2}-q B}{2 q B}-l_{\max }(E)\right)\right. \\
& \left.-\zeta\left(\frac{1}{2}, \frac{E^{2}-m_{\pi}^{2}-q B}{2 q B}+1\right)\right\} .
\end{aligned}
$$

[1] J. Gasser and H. Leutwyler, Phys. Lett. 125B, 325 (1983).

[2] G. Colangelo, J. Gasser, and H. Leutwyler, Phys. Lett. B 488, 261 (2000).

[3] A. Gomez Nicola, F. J. Llanes-Estrada, and J. R. Pelaez, Phys. Lett. B 550, 55 (2002).

[4] N. Kaiser, Phys. Rev. C 59, 2945 (1999).

[5] E. Quack, P. Zhuang, Y. Kalinovsky, S. P. Klevansky, and J. Hufner, Phys. Lett. B 348, 1 (1995).

[6] Y. B. He, J. Hufner, S. P. Klevansky, and P. Rehberg, Nucl. Phys. A630, 719 (1998).

[7] M. Loewe and C. Martinez, Phys. Rev. D 77, 105006 (2008).

[8] J. R. Peláez, Phys. Rep. 658, 1 (2016).

[9] F. Divotgey, P. Kovacs, F. Giacosa, and D. H. Rischke, Eur. Phys. J. A 54, 5 (2018).

[10] B. Peyaud, Nucl. Phys. B, Proc. Suppl. 187, 29 (2009).

[11] S. Weinberg, Phys. Rev. Lett. 17, 616 (1966).

[12] S. Mao, Phys. Rev. D 99, 056005 (2019).

[13] M. Loewe, L. Monje, E. Muñoz, A. Raya, and R. Zamora, Phys. Rev. D 99, 056002 (2019).

[14] B. Lee, Chiral Dynamics (Gordon and Breach, New York, 1972).

[15] A. Ayala, R. L. S. Farias, S. Ortiz, L. A. Hernandez, D. Manreza Paret, and R. Zamora, Phys. Rev. D 98, 114008 (2018).

[16] A. Ayala, M. Hentschinski, L. A. Hernandez, M. Loewe, and R. Zamora, Phys. Rev. D 98, 114002 (2018).

[17] A. Ayala, C. A. Dominguez, L. A. Hernandez, M. Loewe, and R. Zamora, Phys. Rev. D 92, 096011 (2015).

[18] M. Loewe, C. Villavicencio, and R. Zamora, Phys. Rev. D 89, 016004 (2014).
[19] A. Larsen, Z. Phys. C 33, 291 (1986).

[20] N. Bilic and H. Nikolic, Eur. Phys. J. C 6, 515 (1999).

[21] H. Mao, N. Petropoulos, and W.-K. Zhao, J. Phys. G 32, 2187 (2006); N. Petropoulos, arXiv:hep-ph/0402136 and references therein.

[22] B. J. Schaefer and M. Wagner, Phys. Rev. D 79, 014018 (2009).

[23] P. Kovacs and Z. Szep, Phys. Rev. D 77, 065016 (2008).

[24] P. Kovacs and Z. Szep, Phys. Rev. D 93, 114014 (2016).

[25] J. Gasser and H. Leutwyler, Ann. Phys. (N.Y.) 158, 142 (1984).

[26] P. D. B. Collins, An Introduction to Regge Theory on High Energy Physics (Cambridge University Press, Cambridge, England, 1977).

[27] L. Rosellet et al., Phys. Rev. D 15, 574 (1977).

[28] B. Adeva et al., Phys. Lett. B 704, 24 (2011).

[29] X.-H. Liu, F.-K. Guo, and E. Epelbaum, Eur. Phys. J. C 73, 2284 (2013).

[30] J. R. Batley et al. (NA48/2 Collaboration), Eur. Phys. J. C 54, 411 (2008); 70, 635 (2010); Phys. Lett. B 633, 173 (2006); 686, 101 (2010).

[31] S. Pislak et al. (BNL-E865 Collaboration), Phys. Rev. Lett. 87, 221801 (2001); 105, 019901(E) (2010); Phys. Rev. D 67, 072004 (2003); 81, 119903(E) (2010).

[32] M. Loewe, L. Monje, and R. Zamora, Phys. Rev. D 97, 056023 (2018).

[33] M. Loewe and C. Martínez, Phys. Rev. D 77, 105006 (2008); 78, 069902 (2008).

[34] A. Ayala, A. Sanchez, G. Piccinelli, and S. Sahu, Phys. Rev. D 71, 023004 (2005).

[35] M. Loewe and J. Ruiz, Phys. Rev. D 78, 096007 (2008). 